\title{
SCREENING AND IDENTIFICATION OF DRUG TARGETS AND VACCINE CANDIDATES FOR HELICOBACTER PYLORI STRAIN Hp26695
}

\author{
Amita Martin Corolina Pasupuleti, Deepthi Nammi and Nageswara Rao Reddy Neelapu* \\ Department of Bioinformatics, School of Life Sciences, GITAM Institute of Science, GITAM University, \\ Rushikonda campus, Visakhapatnam - 530045 (AP), India.
}

DOI: http://dx.doi.org/10.24327/ijrsr.2017.0804.0140

\begin{tabular}{l}
\hline ARTICLE INFO \\
\hline Article History: \\
Received $15^{\text {th } J a n u a r y, ~} 2017$ \\
Received in revised form $25^{\text {th }}$ \\
February, 2017 \\
Accepted $28^{\text {th }}$ March, 2017 \\
Published online $28^{\text {th }}$ April, 2017 \\
\hline
\end{tabular}

\begin{abstract}
Helicobacter pylori, a class 1 carcinogen colonizes stomach causing gastric carcinoma. Rising antibiotic resistance and reinfections are drawbacks of antibiotic therapies. Alternating drugs and vaccination may be the promising approach to prevent and treat reoccurring infections. Therefore, there is a need for discovery of drug targets, drugs and vaccine candidates for the treatment of $H$. pylori. An objective of this current study is to identify potential drug targets and suitable vaccine candidates for $H$. pylori strain $H p 26695$ by insilico genome and proteome analysis.

Drug targets were identified initially by comparing the genomes between $H$. pylori and Homosapien sapiens using RAST. RAST identified a total of 569 unique genes. These unique genes later were subjected to non-homology and gene property analysis to identify the potential drug targets. BLASTpfollowed by gene property analysis of 569 unique genes identified seven potential drug targets.

Vaccine candidates were identified initially by screening protein sequences for pathogenic factors. These pathogenic factors were screened to identify non-homologous molecules and secondary structure patterns (helices). The proteins $\leq 3$ helices are subjected to screening of antigenic nature followed by allergenicity. The proteins qualifying the above criteria were screened for antigens, Bcells and T-cell epitopes. Proteins showing positive predictions for antigenic, B-cell, T-cell activities are thus shortlisted as vaccine candidates for vaccine designing. Analysis identified 16 immunogenic proteins contributing to immune-response. These methods have enabled rapid identification of potential drug targets and vaccine candidates for strain Hp26695 with possible therapeutic implications for gastric cancer. cancer.
\end{abstract}

Copyright (C) Amita Martin Corolinaet al, 2017, this is an open-access article distributed under the terms of the Creative Commons Attribution License, which permits unrestricted use, distribution and reproduction in any medium, provided the original work is properly cited.

\section{INTRODUCTION}

Gastric cancer is caused by infection of class 1 carcinogen Helicobacter pylori (Zhang, 1994). Treatment for H. pylori infection includes drugs to relieve from pain and acidity, but not for gastritis, peptic ulcers, and gastric cancer. Carcinogenic activity of $H$. pylori suggests the need for discovery of new drug targets and drugs for prevention of $H$. pylori. Laboratory techniques and bioinformatics approaches are used to identify drug targets which can influence growth, colonization and virulence of $H$. pylori(Neelapuet al., 2014). Availability of the complete $H$. pylori genome sequence of pathogens provides us the platform and opportunity to mine the genome and harness the potential drug targets. Comparative genomic analysis between host and pathogen would provide us with a tremendous amount of information that can be useful in drug target identification (Neelapuet al., 2013).Comparative genomics analysis of host with pathogens revealed potential drug targets in Staphylococcus aureus (Uddinet al., 2014), $H$. pylori (Neelapu and Pavani, 2013; Neelapuet al., 2015;Nammiet al., 2016)Listeria monocytogenes(Hossainet al., 2013), Leishmaniainfantum(Sutharet al., 2009), L. major (Florezet al., 2010), Mycobacterium leprae(Wiwanitkit, 2014), Pseudomonas aeruginosa(Sakharkaret al., 2004), Schistosomamansomi(Caffreyet al., 2009).Metabolic pathway analysis (Sarkaret al., 2012), reverse docking (Caiet al., 2006) and screening for essential genes (Duttaet al., 2006) are used to identify drug targets in H. pylori. However, there are no specific reports to date, on comparing genomes of $H$. pylori strain Hp26695 with host Homosapiens to identify drug targets in H. pylori. Therefore, comparing genome of host and pathogen may provide novel drug targets for $H$. pylori strain Hp26695. 
Rising antibiotic resistance due to efflux pumps (Rauws and Tytgat, 1990; Graham et al., 1991), potential reinfection of $H$. pylori even after successful eradication therapy (Arora and Czinn, 2005), inhibition of T-cell stimulation by vacuolatingcytotoxin (Vac A) (Molinari, 1998; Reyrat, 1999), and the highly inflammatory nature by the constituents of the cell wall suggests vaccination as an alternative for protection against $H$. pylori. For better immunization the half-life of the antimicrobial agents should be long enough to be effective and also penetrate mucosal barrier (Arora and Czinn, 2005). Hence, the use of vaccines with appropriate immunogens may provide immune protection against $H$. pylori. Therefore, mining the genomic sequences via bioinformatics approaches for immunological data would provide suitable vaccine candidates. The objective of the current paper is to screen and identify novel drug targets and vaccine candidates for therapeutic intervention of $H$. pylori.

\section{MATERIAL AND METHODS}

\section{Sample}

Complete genomeof $H$. pylori strain Hp26695 with the geographical origin of Europe has the following accession number and genome length NC 018939 and 1,667,867 bp respectively (Manolovet al., 2014). In our study, identification of novel drug targets and vaccine candidates for $H$. pylori has been accomplished for the first time in $H$. pylori strain Hp26695. Novel drug targets were screened and identified using an integrated approach of genome, proteome and metabolic pathway analysis followed by primary property analysis of the genes/proteins using computational resources. Novel vaccine candidates were screened and identified by searching for pathogenic factors, followed by non-homology, secondary patterns and subsequent analysis for antigens, nonallergens and epitopes using computational resources.

\section{Screening and Identification of Drug Targets for $\mathrm{H}$. pylori}

The following protocol was followed for screening and identification of novel drug targets in H. pylori (Figure 1)

\section{Drug target screening for identification of unique molecules in $\mathrm{H}$. pylori}

Comparative genome analysis was performed to screen the drug targets for pathogen $H$. pylori.Genomeof $H$. pylori strain Hp26695 was initially annotated and further reconstructed for metabolic pathways using Rapid Annotation Subsystem Technology (RAST) server (Aziz et al., 2008). Comparative genome analysis between pathogen $H$. pylori and host Homo sapiens sapienswas performed using RAST to screen unique genes that are only present in pathogen and not present in the host (Table 1). Genes which are unique to $H$. pylori in the above method were filtered and catalogued.

\section{Drug target screening for confirmation of unique molecules in H. pylori}

Bacterial genes which are non-homologous to humans are essential for pathogen. To identify the non-homologous molecules in $H$. pylori, homology at the level of sequence and structure of molecules were used as the parameters. BLASTp (Altschulet al., 1990) which is based on principle of homology was used to confirm the uniqueness of the catalogued genes in
H. pylori by comparing genes against Homosapiens sapiens (Table 1).

\section{Drug target identification}

A set of computational resources were used to analyse the characteristic features of the genes, to identify the potential drug targets.BTXpred (Saha and Raghava, 2007), SRTpred (Garg and Raghava, 2008), VGIchan (Saha et al., 2007) and VICMpred (Saha and Raghava, 2006) are the potential targets servers (Table 1) to identify the potentiality of the drug targets. Catalogued genes were verified for their potentiality as drug targets using the above list of servers.

\section{Screening and Identification of Vaccine Candidates for $\boldsymbol{H}$. pylori}

The following protocol was followed for screening and identification of vaccine candidates in H. pylori (Figure 2).

\section{Screening of proteome for identification of pathogenic factors in $\mathrm{H}$. pylori}

The bacterial genome was retrieved and the translated protein sequences of the pathogen are screened for pathogenic factors. Virulence factors, secretory proteins, outer-membrane proteins, bacterial toxins are the pathogenic factors. VirulentPred, EffectiveDB, CELLO, BTXpred (Table 1) are used to screen virulence factors, secretory proteins, outer-membrane proteins and bacterial toxins respectively. Further, the pathogenic factors of the bacteria are screened for non-homologous proteins as per the procedure described above in "Drug target screening for confirmation of unique molecules in H. pylori".

\section{Screening of non-homologous proteins for identification of secondary patterns in $\mathrm{H}$. pylori}

The non-homologous proteins of the bacteria are screened for secondary patterns - helices using Chou Fasman method by CFSSP: Chou and Fasman Secondary Structure Prediction Server (Ashok Kumar, 2013) (Table 1). Proteins with alphahelices $\leq 3$ are selected for further analysis.

\section{Predicting function of proteome}

Metabolic pathway analysis using RAST (Aziz et al., 2008), BLASTp (Altschulet al., 1990), NCBI Conserved Domain Database (Marchler-Baueret al., 2004), ProtFun 2.2 Server (Jensen et al., 2002) (Table 1) and literature-search are used for identifying the function of proteins.

\section{Screening of proteome for antigens}

The proteins fulfilling the above criteria are screened for antigens using Antigenic Emboss Server (Kolaskar and Tongaonkar, 1990) (Table 1). These proteins are catalogued and subjected to further analysis.

\section{Screening of proteome for non-allergenicity}

The proteins which are antigenic in nature are screened for allergenicity using server Allergen Online (Maria et al., 2006) (Table 1). The non-allergens are shortlisted and catalogued for further analysis. 
Table 1Computational resources used for identification of potential drug targets and suitable vaccine candidates in Helicobacter pylori

\begin{tabular}{|c|c|c|c|}
\hline S. No & $\begin{array}{l}\text { Server } \\
\text { Name }\end{array}$ & Server Function & Reference \\
\hline 1 & RAST & $\begin{array}{l}\text { Rapid Annotation Subsystem Technology Server is used for prediction of unique genes } \\
\text { based on metabolic pathways. }\end{array}$ & Aziz et al., (2008) \\
\hline 2 & BTXpred & $\begin{array}{c}\text { Server is for prediction of bacterial toxins and its function from primary amino acid } \\
\text { sequence. }\end{array}$ & Saha and Raghava, (2007) \\
\hline 3 & SRTpred & Server classifies protein sequence as secretory or non-secretory proteins. & Garg and Raghava, (2008) \\
\hline 4 & VGIchan & $\begin{array}{l}\text { Server predicts voltage gated ion-channels and classifies them into sodium, potassium, } \\
\text { calcium and chloride ion channels from primary amino acid sequences. }\end{array}$ & Sahaet al., (2007) \\
\hline 5 & VICM pred & $\begin{array}{l}\text { Server aids in broad functional classification of bacterial proteins into virulence factors, } \\
\text { information molecule, cellular process and metabolism molecule. }\end{array}$ & Saha and Raghava, (2006) \\
\hline 6 & VirulentPred & $\begin{array}{l}\text { VirulentPred predicts virulence proteins using reliable Support Vector Machine (SVM) } \\
\text { algorithm. This server has a prediction accuracy of } 65 \% \text {. }\end{array}$ & Garget al., (2008) \\
\hline 7 & EffectiveDB & $\begin{array}{l}\text { EffectiveDB predicts putative effectors by identifying eukaryotic-like protein domains and } \\
\text { by detecting the } 2 \text { known types of signal peptides. This server has a prediction accuracy of } \\
\qquad 80 \% \text {. }\end{array}$ & Jehlet al., (2011) \\
\hline 8 & CELLO-V & $\begin{array}{c}\text { CELLO (subcellular localization predictor) predicts protein present in outer membrane } \\
\text { directly from protein sequences. The server uses two-level support vector machine (SVM) } \\
\text { system: the first level contains SVM classifiers and the second level SVM classifier } \\
\text { function to generate the probability distribution of decisions for possible localizations. } \\
\text { This server has a prediction accuracy of } 90 \% \text {. }\end{array}$ & Yu et al., (2004) \\
\hline 9 & $\begin{array}{l}\text { CFSSP } \\
\text { Server }\end{array}$ & $\begin{array}{c}\text { Chou \&Fasman Secondary Structure Prediction (CFSSP) server predicts protein } \\
\text { conformation like helices, beta sheets, random coils based on Chou \&Fasman algorithm. } \\
\text { This server has a prediction accuracy of } 88 \% \text {. }\end{array}$ & Ashok Kumar, (2013) \\
\hline 10 & Antigenic & $\begin{array}{l}\text { Antigenic server predicts potentially antigenic sites of a protein sequence. The server uses } \\
\text { semi-empirical method consisting physicochemical properties of amino acids and their } \\
\text { frequencies of occurrence in experimentally known epitopes. This server has a prediction } \\
\text { accuracy of } 75 \% \text {. }\end{array}$ & Kolaskar and Tongaonkar, (1990) \\
\hline 11 & ABCpred & $\begin{array}{l}\text { ABCpred server predicts B-cell epitope using Recurrent Artificial Neural Network- } \\
\text { (ANN-) based algorithm. This server has a prediction accuracy of } 65.93 \% \text {. } \\
\text { HLApred server identifies the experimentally proven binders taken from MHCBN }\end{array}$ & Saha and Raghava, (2006) \\
\hline 12 & HLApred & $\begin{array}{c}\text { database based on quantitative matrices HLA alleles which were obtained from literature. } 1 \\
\text { This server has a prediction accuracy of } 80 \% .\end{array}$ & vww.imtech.res.in/raghava/hlapred/index.html \\
\hline 13 & $\begin{array}{l}\text { Allergen } \\
\text { Online }\end{array}$ & $\begin{array}{l}\text { Cross reactive allergens are predicted using server Allergen Online based on BLOSUM50 } \\
\text { scoring matrix algorithm. This Server has a prediction accuracy of } 70 \% \text {. }\end{array}$ & Maria et al., (2006) \\
\hline
\end{tabular}

Figure1 Screening and identification of novel drug targets for $H$. pylori

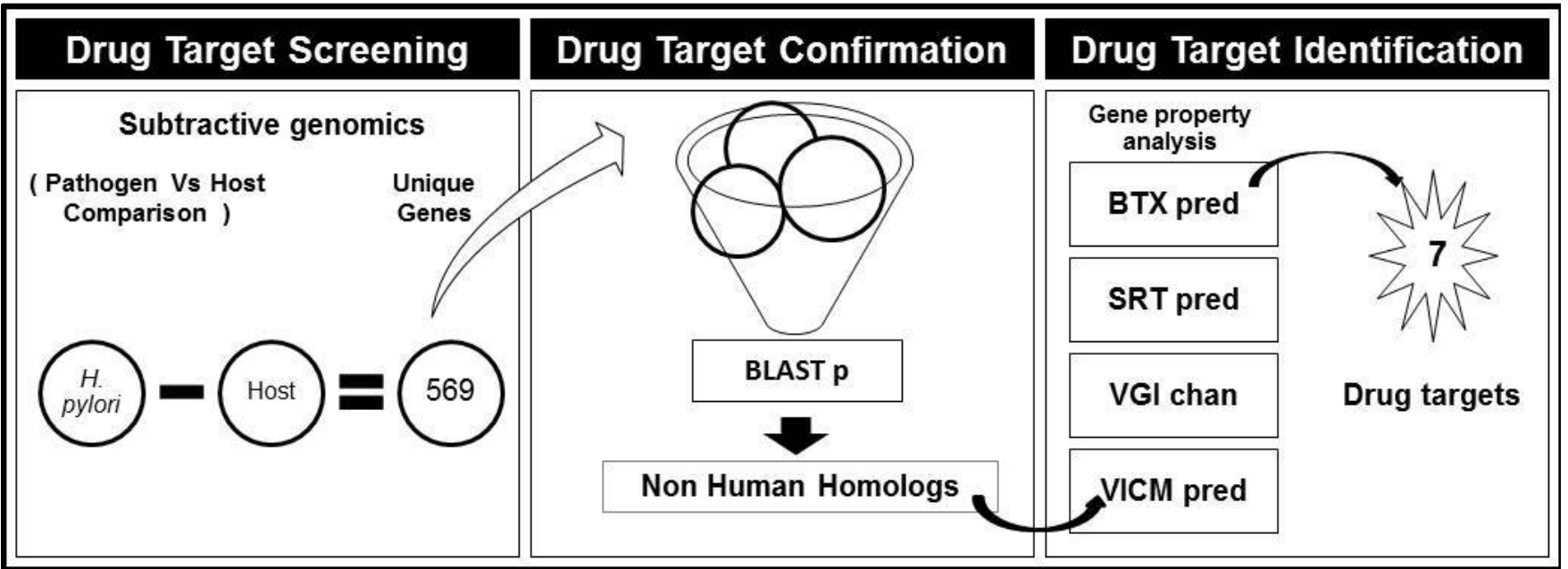

Screening of non-allergenic proteome for identification of antigenic and epitope regions in $\mathrm{H}$. pylori

The proteins fulfilling the above criteria are screened for promising epitopes which include both $\mathrm{B}$ cell \& $\mathrm{T}$ cell epitopes. B-cells epitopes are screened and identified using ABCpred Server (Saha et al., 2006) (Table 1), whereas T-cell epitopes are screened and identified using HLApred (Table 1). Finally, proteins satisfying the above three criteria's i.e. proteins showing positive predictions for antigenic, B-cell, Tcell activities are short listed as vaccine candidates for vaccine designing.

\section{RESULTS}

Genome Wide InsilicoAnalysis for Screening of Drug Targets in $\mathrm{H}$. pylori

Genome wide in silico analysis for screening drug targets identified 569 unique genes in H. pylori strain Hp26695 (Table 2 ). These molecules fall under 24 metabolic categories as shown in Table 2. Proteome analysis followed by gene property analysis of 569 unique genes identified seven potential drug targets in $H$. pylori (Table 3). These molecules fall under five metabolic categories as shown in Table 3. 
Proteome Wide InsilicoAnalysis for Screening of Vaccine Candidates in $\mathrm{H}$. pylori

Screening of 1469 proteins in H. pylori strain Hp26695 identified 643 pathogenic factors.VirulentPred, EffectiveDB, CELLO, BTXpredidentified 399, 291, 197, 18 proteins respectively (Table 4). Analysis of 643 pathogenic factors identified 146 non-homologous proteins (Table 4). Screening of 146 non-homologous proteins for secondary structure patterns identified 46 proteins with $\leq 3$ helices (Table 4 ). Analysis of these 46 proteins identified 44 proteins which are antigenic in nature. Further analysis of 44 antigenic proteins identified 29 non-allergenic proteins (Table 4). Analysis of these 29 non-allergenic proteins revealed antigens (99), B-cell (198) and T-cell (419) epitopes shortlisting to 16 proteins with antigenic regions (26) and potential epitopes (52) (Table 5, 6, $7,8)$. These 16 immunogenic proteins contribute to immuneresponse and are well-suited for vaccine designing (Table 8). Insilicoscreening of peptides have helped examining the molecular properties, further in vivo-studies would be most helpful in bringing out potentially specific vaccine candidates.
Table 2Total number of unique genes identified for each metabolic category using RAST in $H$. pylori strain $H p 26695$ by comparing genomes of pathogen $H$. pylori and HostHomosapiens sapiens

\begin{tabular}{ccc}
\hline S. No & Metabolic category & Hp26655 \\
\hline 1 & Amino Acids and Derivatives & 49 \\
2 & Carbohydrates & 37 \\
3 & Cell Division and Cell Cycle & 3 \\
4 & Cell Wall and Capsule & 43 \\
5 & Clustering-based subsystems & 82 \\
6 & Cofactors, Vitamins, Prosthetic Groups, & 50 \\
7 & Pigments & 31 \\
8 & DNA Metabolism & 29 \\
9 & Fatty Acids, Lipids, and Isoprenoids & 22 \\
10 & Membrane Transport & 14 \\
11 & Miscellaneous & 38 \\
12 & Motility and Chemotaxis & 2 \\
13 & Nucleosides and Nucleotides & 3 \\
14 & Phosphorus Metabolism & 3 \\
15 & Potassium metabolism & 84 \\
16 & Protein Metabolism & 18 \\
17 & RNA Metabolism & 7 \\
18 & Regulation and Cell signalling & 28 \\
19 & Respiration & 15 \\
20 & Stress Response & 2 \\
21 & Sulfur Metabolism & 9 \\
22 & Virulence, Disease and Defense & 0 \\
& Iron acquisition and metabolism & 569 \\
\hline
\end{tabular}

Figure 2Screening and identification of vaccine candidates for $H$. pylori

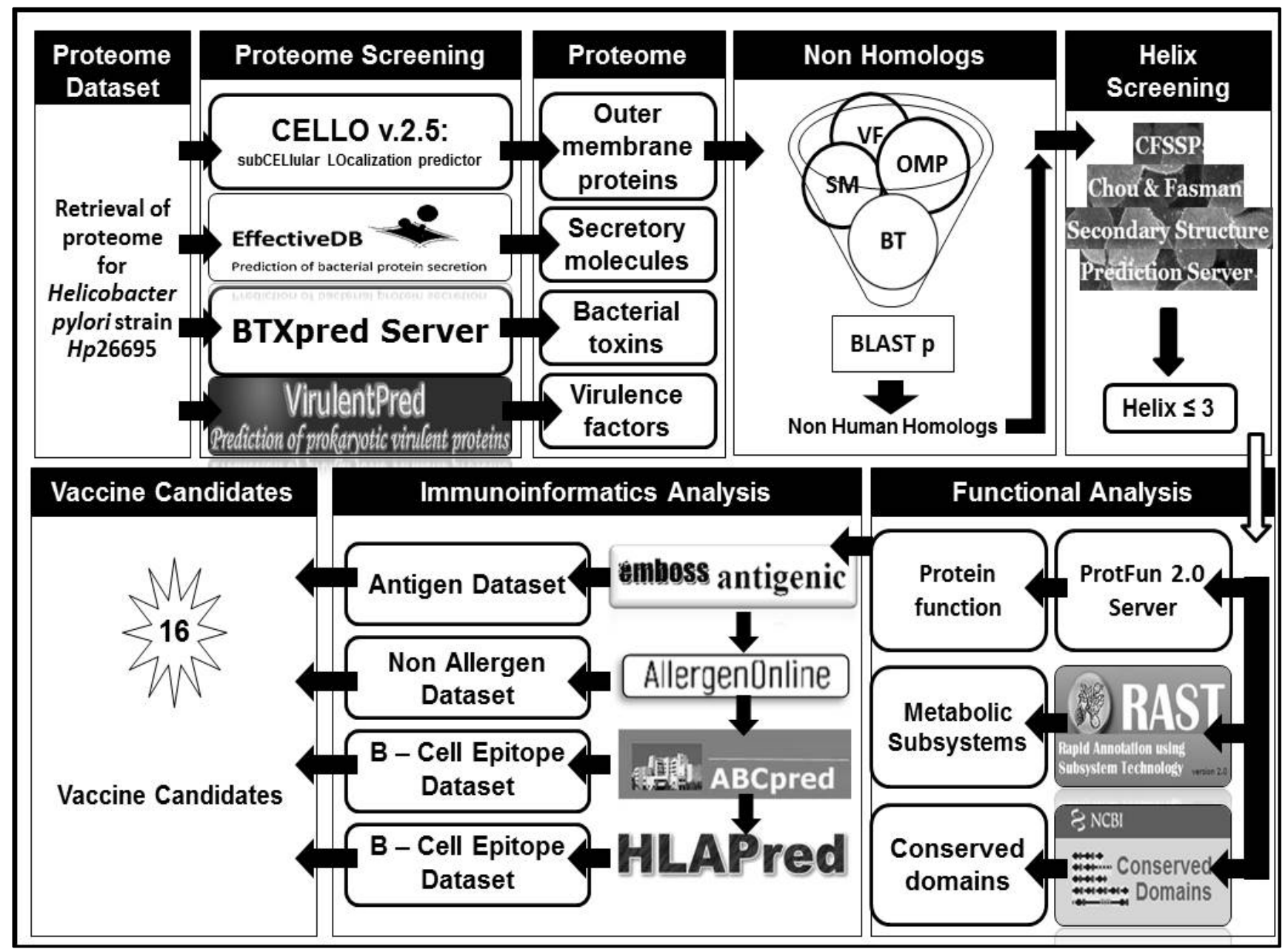


Table 3Drug targets identified in the H. pylori strain Hp26695 by comparing genomes of pathogen H. pylori and HostHomosapiens sapiens

\begin{tabular}{|c|c|c|c|c|c|}
\hline S. No & Drug Target & Metabolic Category & Gene ID & Validated $^{1}$ & Novel $^{2}$ \\
\hline 1 & Menaquinone via futalosine step 1 & $\begin{array}{l}\text { Cofactors, Vitamins, Prosthetic } \\
\text { Groups, Pigments }\end{array}$ & GI:15645397 & + & \\
\hline 2 & Type III restriction-modification system methylation subunit & DNA Metabolism & GI:15645218 & + & \\
\hline 3 & Dipeptide transport system permease protein DppB & Membrane Transport & GI:15644927 & + & \\
\hline 4 & Dipeptide transport system permease protein DppC & Membrane Transport & GI:15644928 & + & \\
\hline 5 & $\begin{array}{l}\text { Ferric siderophore transport system, biopolymer transport } \\
\text { protein ExbB }\end{array}$ & Membrane Transport & GI:15646054 & & + \\
\hline 6 & Ribonuclease BN & RNA Metabolism & GI:15646017 & & + \\
\hline 7 & NADH-ubiquinone oxidoreductase chain $\mathrm{J}$ & Respiration & GI: 15645883 & & + \\
\hline
\end{tabular}

${ }^{1}$ Experimentally validated drug targets either by genetically or biochemically

${ }^{2}$ Novel drug targets identified in this study

Table 4 Pathogenic factors, non-homology proteins, helices, antigens and non-allergens identified in H. pylori strain Hp26695

\begin{tabular}{cccccc}
\hline Analysis Type & Virulence Factor & Secretory Proteins & $\begin{array}{c}\text { Outer Membrane } \\
\text { Proteins }\end{array}$ & $\begin{array}{c}\text { Bacterial } \\
\text { Toxin }\end{array}$ & $\begin{array}{c}\text { Total Proteins } \\
\text { Pathogenic factors }\end{array}$ \\
\hline Non-homology to humans & 89 & 291 & 197 & 643 \\
Proteins with $\leq 3$ helices & 37 & 71 & 57 & 3 & 18 \\
Antigenic proteins & 35 & 17 & 4 & 0 & 0 \\
Non-allergenic proteins & 24 & 16 & 3 & 46 & 0 \\
\hline
\end{tabular}

\section{DISCUSSION}

Insilico methods helped in identifying novel drug targets and vaccine candidates for $H$. pylori. This was the first report on implementation of insilico subtractive genomics and insilico reverse vaccinology to identify novel drug targets and vaccine candidates respectively for $H$. pylori strain $H p 26695$.

\section{Drug Targets for H. pylori}

Subtractive genomics, metabolic pathway analysis, essential gene analysis and reverse docking were earlier used to identify drug targets for $H$. pylori. Cai et al. (2006) used reverse docking to identify a drug target in $H$. pylori. Dutta et al. (2006) identified 40 essential genes as drug targets in $H$. pyloriHpAG1 strain. Sarkhar et al. (2012) identified lipopolysaccharide biosynthesis pathway as a source of potential drug targets in $H$. pylori using metabolic pathway analysis. Neelapu and Pavani (2013) identified 17 novel drug targets in $H p \mathrm{~B} 38, H p \mathrm{P} 12, H p \mathrm{G} 27, H p$ Shi470, HpSJM180 strains of $H$. pylori using genomics and proteomics. Neelapu et al. (2015)using genomics and proteomics identified 29 novel drug targets in HpAG1 strain of H. pylori. Nammi et al., (2016) using comparative genomics identified 29 novel drug targets. In this present study subtractive genomics was used to identify novel drug targets in addition to the existing drug target's pool of H. pylori (Neelapuet al., 2016). Mining the genome of pathogen has identified nearly seven potential drug targets for H. pylori. These novel drug targets fall under the following categories of functions such as Cofactors, Vitamins, Prosthetic Groups, and Pigments; DNA Metabolism; Membrane Transport; RNA Metabolism; and Respiration.

Drug targets influencing cofactors, vitamins, prosthetic groups, pigments of the pathogen

Menaquinone via futalosine step 1, is identified as the drug target in $H$. pylori. This drug target influences metabolic pathway of cofactors, vitamins, prosthetic groups, pigments of the pathogen. Menaquinone is an important component of the electron transfer pathway. An alternative pathway is present in the human commensal intestinal bacteria H. pylori and
Campylobacter jejuni. Disruption of menaquinone via futalosine pathway had shown inhibition of bacteriostatic growth (Arakawa et al., 2011). Therefore, designing an inhibitor for menaquinone via futalosine step 1 would affect the growth of $H$. pylori.

\section{Drug targets influencing DNA metabolism of the pathogen}

Type III restriction-modification system methylation subunit of restriction-modification (R-M) systems, is identified as the drug target in $\mathrm{H}$. pylori. This drug target influences metabolic pathway DNA metabolism of the pathogen. H. pylori are naturally competent and prone to take DNA from the environment (Dorer et al., 2010) and bacteriophages also infect H. pylori (Heintschel et al., 1993). Missense and frameshift mutations can accumulate and inactivate genes when bacteriophages or free DNA or plasmids enter into other cells.

Evidence is there that sometimes even both endonuclease and methylase genes of R-M systems have to be turned off. However, $H$. pylori in a population have a very good defensive system, where R-M systems protect the genome of $H$. pylori from accumulated mutations when bacteriophages or free DNA or plasmids enter into other cells. Mutant strains lacking this display a pleiotropic phenotype, including increased mutability, hyper recombination, and increased sensitivity to DNAdamaging agents. Therefore, designing an inhibitor for type III restriction-modification system methylation subunit decreases the rate of survival of $H$. pylori due to gross changes occurring in the genetic material.

\section{Drug targets influencing DNA metabolism of the pathogen}

Dipeptide transport system permease protein DppB, dipeptide transport system permease protein $\mathrm{DppC}$ and ferric siderophore transport system, biopolymer transport protein ExbB are identified as drug targets in $H$. pylori. These drug targets influence membrane transport of the pathogen.

Dipeptide $D p p A B C D F$ and oligopeptideopp $A B C D$ genes are a class of ABC-type transporter in H. pylori. Dipeptide transport system permease protein - DppBC are responsible for transporting dipeptides. Dipeptide and oligopeptide system 
Table 5Antigens predicted in the proteome of $H$. pylori strain Hp26695 using Antigenic server

\begin{tabular}{|c|c|c|c|c|}
\hline S. No & Protein ID & Protein Name & Antigen No & Antigen Sequence \\
\hline \multirow{3}{*}{1} & \multirow{3}{*}{ NP_206874.1 } & \multirow{3}{*}{ Lipoprotein signal peptidase } & AG1 & KSLLVFMGVFFLIFGVDQAIKYAILEG \\
\hline & & & AG2 & YESLMIDIVLVFNKGVAFSLLSFLEGGLKYLQILLILGLFIFLM \\
\hline & & & AG3 & $\begin{array}{l}\text { GAGVSNVLDRFVHGGVVDYVYYHYGFDFAIFNFADVMIDVGVGVLL } \\
\text { LKQFFFK }\end{array}$ \\
\hline 2 & NP 206881.1 & Membrane Protein & AG4 & ALKSKAFRVSIQWNALVRKLLALE \\
\hline 3 & $\mathrm{NP}_{-} 206885.1$ & Cytochrome $\mathrm{c}$ oxidase VI a. & AG5 & IAKKAVKIVFFLGLVVVLLMMI \\
\hline \multirow{2}{*}{4} & \multirow{2}{*}{ NP_206945.1 } & \multirow{2}{*}{ CBB3-type cytochrome c oxidase Q } & AG6 & LRGFAYAFFTILFTLFLYAYIFSM \\
\hline & & & AG7 & YGYLALND \\
\hline \multirow[t]{5}{*}{5} & \multirow{2}{*}{ NP_206947.1 } & \multirow{2}{*}{ DUF4006 superfamily } & AG8 & GNLLIVVILLCVAVFFTLKAIHIQK \\
\hline & & & AG9 & YELVNQ \\
\hline & & & AG10 & LHFSHL \\
\hline & \multirow{6}{*}{ NP_207071.1 } & & AG11 & DVGFIIKNLVFLGVFSLLGW \\
\hline & & & AG12 & FLWPSMLELKKILLE \\
\hline \multirow[t]{7}{*}{6} & & Translation Protein & AG13 & KSVLEYAQR \\
\hline & & & AG14 & ESLLKI \\
\hline & & & AG15 & LEKILKKCFDAYKIKPLLSQNS \\
\hline & & & AG16 & KTQFFIMA \\
\hline & & & AG17 & KTYLFFTLINKYLPSAQSQLPLKIS \\
\hline & & & AG18 & KLLVLEFR \\
\hline & & & AG19 & WMYSTFISLKTHLQFIE \\
\hline & NP 2071251 - & & AG20 & HRYFLF \\
\hline 7 & $\mathrm{NP}_{-} 20 / 125.1$ & Flagellar protein FlaG & AG21 & EEGVYLGVGSI \\
\hline & & & AG22 & KHGYLGIYKNP \\
\hline & & & AG23 & ILKALEFI \\
\hline & & & AG24 & FEEFQLHSLHLEV \\
\hline & & & AG25 & FIDVLLYYK \\
\hline 8 & NP 207138.1 & Tranclation Drotain & AG26 & LKLEGCEKHCKKKYAIEKVIKEVGLELKSKSVMPY \\
\hline & & Iranstation Protem & AG27 & RSQIISILMK \\
\hline 9 & NP_207241.1 & & AG28 & SLAILMPSFLLAAPDYK \\
\hline & & Transporter Protein & AG29 & KFTQILDFI \\
\hline & & & AG30 & IKAIGGLIIVGTCIYAY \\
\hline & & & AG31 & WKCVGIIIITAAIS \\
\hline 10 & NP 207255.1 & & AG32 & VGISVSNL \\
\hline & & Iranslation Protein & AG33 & FLWLNAKSFLLSGFVPFIMIPWLDILNSFVLYVCFLLIFSIAE \\
\hline & & & AG34 & SDILIAHSK \\
\hline & & & AG35 & SLIFKKVRIYSKMLVALGLSSVLIGCAM \\
\hline & & & AG36 & SSEHVTPLDFNYPIHIVQAPQNHHVVGILTPRIQVSDNLKPYID \\
\hline & & & AG37 & FQDALINQIQT \\
\hline 11 & NP_207289.1 & putative paralog of $H p \mathrm{aA}$ & AG38 & RGYQVLR \\
\hline & & & AG39 & KIFSVLDLKGWVGILE \\
\hline & & & AG40 & LDTLVDQSSGSVWF \\
\hline & & & AG41 & SNRVVHDFAVEVGT \\
\hline & & & AG42 & NRMYAVVMK \\
\hline 12 & NP 2073321 & & AG43 & ISKLKQNFLQFKH \\
\hline 12 & NP_20/332.1 & Cag pathogenicity island protein cag 15 & AG44 & $\begin{array}{l}\text { LDKYSLYYRLFNISSIVIGFLVALFSYGAGVILVYPILFLFALIIKPSFFYY } \\
\text { TTYLLLLVSLSIISKYYLLSHA }\end{array}$ \\
\hline & & & AG45 & KLIILMT \\
\hline & & & AG46 & HPEYIPCKVTCVTSG \\
\hline 13 & NP_207346.1 & 50 S ribosomal protein $\mathrm{L} 31$ & AG47 & EIEVLST \\
\hline & & & AG48 & ISSFCHPFY \\
\hline 14 & & & AG49 & RNLVVTEC \\
\hline 14 & NP_207357.1 & $30 \mathrm{~S}$ ribosomal protein S21 & AG50 & KKKVLKRLYML \\
\hline 15 & NP_207389.1 & Transport and binding protein & AG51 & LGLILSLAAILIAFK \\
\hline 16 & & & AG52 & SLRACFLTLFFSGY \\
\hline & NP 2075311 & Integral membrane protein & AG53 & IGSLVALLLGLPVLIFSANTLFLGAVFVGLIAIAQI \\
\hline & $\mathrm{NP}_{-} 20 / 531.1$ & & AG54 & SSYIVIDEL \\
\hline & & & AG55 & AMAISGLSLAGVILSFIFFRIYDITKPSLIGK \\
\hline 17 & NP 207549.1 & Translation Protein Enzyme & AG56 & YKNVYDDD \\
\hline 18 & $\mathrm{NP}^{-} 207582.1$ & Type I restriction-modification system & AG57 & STVVAEF \\
\hline 19 & NP_207692.1 & & AG58 & AIPSKVIAIKDNVVLLETLGVQRE \\
\hline & & Hydrogenase expression/formation & AG59 & GESVKVGDYVLLHIGYVMSK \\
\hline & & & AG60 & LESIELYQE \\
\hline & & & AG61 & VYLVQSD \\
\hline & & & AG62 & IGLLSK \\
\hline 20 & NP 2077101 & & AG63 & NQSVLIESA \\
\hline & $\mathrm{NP}_{-} 20 / / 10.1$ & $\mathrm{TsaC}$ protein (YrdC domain) required & AG64 & FSTLKSLVRAP \\
\hline & & for threonylcarbamoyladenosine $\mathrm{t}(6) \mathrm{A} 37$ & AG65 & TTFIYPNSKAVRVIRG \\
\hline & & modification in tRNA & AG66 & TLYSTS \\
\hline & & & AG67 & LTQCAYDKE \\
\hline & & & AG68 & ASNLADVIVSDE \\
\hline & & & AG69 & SKIFRLY \\
\hline 21 & NP_207792.1 & Translation Protein & AG70 & AHTLYISE \\
\hline & & Iranslation Protein & AG71 & SVFVQDAIIFYLEY \\
\hline
\end{tabular}




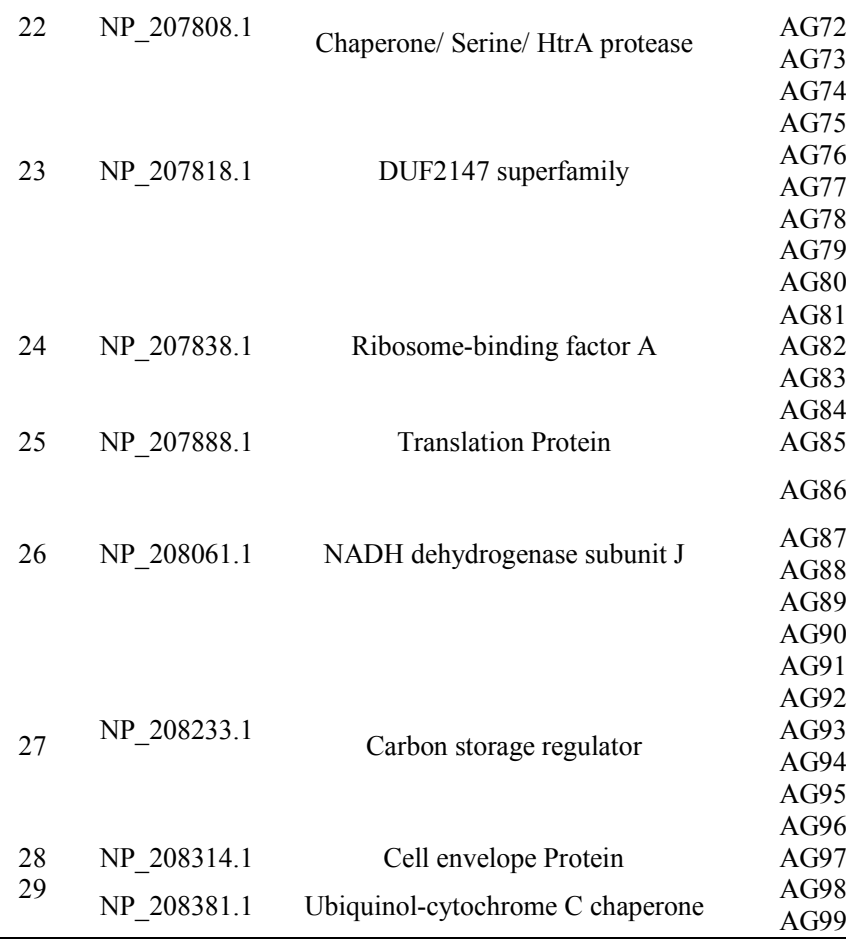

TLFISLALALSLN
KERVSVPSK
VSLVIVFCCFLRAVELPGIY
TQEFLYMKSSFVEF
KFYAYGISDV
KGVVFLSDLIKVGKR
KTYYVRVT
LDEVLKTI
SNLLELLQEALASL
LIRQFVLQAS
WNSLSVTKVECSKGKHHAYVFVLSSILSKL
EKQLRLDAI
KSALLGVRRILGEV
IAFYFFAILTLSMALVVITTTILYAITALASSMVFISAFFFLDAEFLGV
VQITVYVGAVIVMYA
AAEVVERK
PKILCILSFGVALLLTLILSAPS
TGIQKI
EGIVIDDN
HIKVISI
RGSVRLGFE
ESTLILRAE
KEAIVSEN
KASVCVDES
ITHFIAISFVLSLFSACKD
DLEFLKRL
LKDLFDALVYD

Table 6 B-cell, T-cell and Consensus epitopes predicted in the proteome of H. pylori strain Hp26695

\begin{tabular}{|c|c|c|c|c|}
\hline Antigen No & Antigen Sequence & $\begin{array}{c}\text { B-cell Epitopes } \\
(\text { Threshold }>0.60)\end{array}$ & $\begin{array}{c}\text { T-cell Epitopes } \\
\text { (Threshold }>0.70)\end{array}$ & $\begin{array}{c}\text { Consensus } \\
\text { Epitopes } \\
\end{array}$ \\
\hline AG1 & KSLLVFMGVFFLIFGVDQAIKYAILEG & 1 & 3 & - \\
\hline AG2 & YESLMIDIVLVFNKGVAFSLLSFLEGGLKYLQILLILGLFIFLM & 1 & 8 & 1 \\
\hline AG3 & $\begin{array}{l}\text { GAGVSNVLDRFVHGGVVDYVYYHYGFDFAIFNFADVMIDVG } \\
\text { VGVLLLKQFFFK }\end{array}$ & - & 7 & - \\
\hline AG4 & ALKSKAFRVSIQWNALVRKLLALE & 1 & 4 & 2 \\
\hline AG5 & IAKKAVKIVFFLGLVVVLLMMI & - & 5 & - \\
\hline AG6 & LRGFAYAFFTILFTLFLYAYIFSM & 1 & 4 & 2 \\
\hline AG7 & YGYLALND & - & - & - \\
\hline AG8 & GNLLIVVILLCVAVFFTLKAIHIQK & 1 & 6 & 1 \\
\hline AG9 & YELVNQ & - & 1 & - \\
\hline AG10 & LHFSHL & 1 & - & - \\
\hline AG11 & DVGFIIKNLVFLGVFSLLGW & - & - & - \\
\hline AG12 & FLWPSMLELKKILLE & - & - & - \\
\hline AG13 & KSVLEYAQR & - & - & - \\
\hline AG14 & ESLLKI & 1 & - & - \\
\hline AG15 & LEKILKKCFDAYKIKPLLSQNS & - & 1 & - \\
\hline AG16 & KTQFFIMA & - & - & - \\
\hline AG17 & KTYLFFTLINKYLPSAQSQLPLKIS & - & 4 & - \\
\hline AG18 & KLLVLEFR & - & - & - \\
\hline AG19 & WMYSTFISLKTHLQFIE & 2 & 3 & 3 \\
\hline AG20 & HRYFLF & 1 & - & - \\
\hline AG21 & EEGVYLGVGSI & - & - & - \\
\hline AG22 & KHGYLGIYKNP & 1 & - & - \\
\hline AG23 & ILKALEFI & 1 & - & - \\
\hline AG24 & FEEFQLHSLHLEV & 1 & 2 & 1 \\
\hline AG25 & FIDVLLYYK & - & - & - \\
\hline AG26 & LKLEGCEKHCKKKYAIEKVIKEVGLELKSKSVMPY & - & 5 & - \\
\hline AG27 & RSQIISILMK & 1 & - & - \\
\hline AG28 & SLAILMPSFLLAAPDYK & 1 & - & - \\
\hline AG29 & KFTQILDFI & - & - & - \\
\hline AG30 & IKAIGGLIIVGTCIYAY & 1 & 3 & 1 \\
\hline AG31 & WKCVGIIITAAAIS & - & 5 & - \\
\hline AG32 & VGISVSNL & - & 2 & - \\
\hline AG33 & FLWLNAKSFLLSGFVPFIMIPWLDILNSFVLYVCFLLIFSIAE & - & 8 & - \\
\hline AG34 & SDILIAHSK & - & - & - \\
\hline AG35 & SLIFKKVRIYSKMLVALGLSSVLIGCAM & 2 & 3 & 2 \\
\hline AG36 & SSEHVTPLDFNYPIHIVQAPQNHHVVGILTPRIQVSDNLKPYID & 2 & 2 & 2 \\
\hline AG37 & FQDALINQIQT & - & - & - \\
\hline AG38 & RGYQVLR & - & - & - \\
\hline AG39 & KIFSVLDLKGWVGILE & - & - & - \\
\hline
\end{tabular}




\begin{tabular}{|c|c|c|c|c|}
\hline AG40 & LDTLVDQSSGSVWF & 1 & - & - \\
\hline AG41 & SNRVVHDFAVEVGT & 1 & 1 & 1 \\
\hline AG42 & NRMYAVVMK & - & - & - \\
\hline AG43 & ISKLKQNFLQFKH & - & - & - \\
\hline AG44 & $\begin{array}{l}\text { LDKYSLYYRLFNISSIVIGFLVALFSYGAGVILVYPILFLFALIIK } \\
\text { PSFFYYTTYLLLLVSLSIISKYYLLSHA }\end{array}$ & 2 & 5 & 2 \\
\hline AG45 & KLIILMT & - & 1 & - \\
\hline AG46 & HPEYIPCKVTCVTSG & 1 & 1 & 1 \\
\hline AG47 & EIEVLST & 1 & - & - \\
\hline AG48 & ISSFCHPFY & 1 & - & - \\
\hline AG49 & RNLVVTEC & - & - & - \\
\hline AG50 & KKKVLKRLYML & - & - & - \\
\hline AG51 & LGLILSLAAILIAFK & - & - & - \\
\hline AG52 & SLRACFLTLFFSGY & - & - & - \\
\hline AG53 & IGSLVALLLGLPVLIFSANTLFLGAVFVGLIAIAQI & 2 & 8 & 3 \\
\hline AG54 & SSYIVIDEL & - & - & - \\
\hline AG55 & AMAISGLSLAGVILSFIFFRIYDITKPSLIGK & 3 & 6 & 6 \\
\hline AG56 & YKNVYDDD & 1 & - & - \\
\hline AG57 & STVVAEF & 1 & - & - \\
\hline AG58 & AIPSKVIAIKDNVVLLETLGVQRE & 3 & 3 & 1 \\
\hline AG59 & GESVKVGDYVLLHIGYVMSK & 2 & 4 & 4 \\
\hline AG60 & LESIELYQE & - & - & - \\
\hline AG61 & VYLVQSD & - & - & - \\
\hline AG62 & IGLLSK & 2 & - & - \\
\hline AG63 & NQSVLIESA & - & - & - \\
\hline AG64 & FSTLKSLVRAP & 1 & - & - \\
\hline AG65 & TTFIYPNSKAVRVIRG & - & - & - \\
\hline AG66 & TLYSTS & - & 1 & - \\
\hline AG67 & LTQCAYDKE & 1 & - & - \\
\hline AG68 & ASNLADVIVSDE & - & - & - \\
\hline AG69 & SKIFRLY & 1 & - & - \\
\hline AG70 & AHTLYISE & 1 & - & - \\
\hline AG71 & SVFVQDAIIFYLEY & - & 1 & - \\
\hline AG72 & TLFISLALALSLN & - & 2 & - \\
\hline AG73 & KERVSVPSK & 1 & 1 & 1 \\
\hline AG74 & VSLVIVFCCFLRAVELPGIY & 2 & 5 & 4 \\
\hline AG75 & TQEFLYMKSSFVEFF & 2 & 2 & 1 \\
\hline AG76 & KFYAYGISDV & 1 & 1 & 1 \\
\hline AG77 & KGVVFLSDLIKVGKR & - & 3 & - \\
\hline AG78 & KTYYVRVT & - & - & - \\
\hline AG79 & LDEVLKTI & - & - & - \\
\hline AG80 & SNLLELLQEALASL & - & - & - \\
\hline AG81 & LNSLSVTKVECSKGKHHAYVFVLSSDHKILSKL & 2 & 4 & 4 \\
\hline AG82 & LIRQFVLQAS & - & 1 & - \\
\hline AG83 & WFKCPKLSFVSDN & 1 & 1 & 1 \\
\hline AG84 & EKQLRLDAI & 1 & - & - \\
\hline AG85 & KSALLGVRRILGEV & 1 & 2 & - \\
\hline AG86 & $\begin{array}{l}\text { IAFYFFAILTLSMALVVITTTNILYAITALASSMVFISAFFFLLDA } \\
\text { EFLGVVQITVYVGAVIVMYA }\end{array}$ & 3 & 11 & 6 \\
\hline AG87 & AAEVVERK & 1 & - & - \\
\hline AG88 & PKILCILSFGVALLLTLILSAPS & 1 & 2 & 1 \\
\hline AG89 & DAQIPNIKAIGYVLFTNYLIPFEAAALMLLVAMVGGI & 1 & 1 & 1 \\
\hline AG90 & TGIQKI & 1 & - & - \\
\hline AG91 & EGIVIDDN & - & - & - \\
\hline AG92 & HIKVISI & 1 & 1 & 1 \\
\hline AG93 & RGSVRLGFE & - & - & - \\
\hline AG94 & ESTLILRAE & 1 & - & - \\
\hline AG95 & KEAIVSEN & - & - & - \\
\hline AG96 & KASVCVDES & - & - & - \\
\hline AG97 & ITHFIAISFVLSLFSACKD & - & 5 & - \\
\hline AG98 & DLEFLKRL & 1 & - & - \\
\hline AG99 & LKDLFDALVYD & 1 & - & - \\
\hline
\end{tabular}

mutant's in $H$. pylori lacked the ability to use certain dipeptides, hexapeptides, and nonapeptides due to compromisation of either substrate binding domain or permease domains (Weinberg, 2007). Therefore, designing an inhibitor to dipeptide transport system permease protein DppBC would affect the growth and survival of $H$. pylori.

Ferric transport system, biopolymer transport protein ExbB is a member of transporter proteins in $H$. pylori. All bacterial pathogens have developed highly sophisticated iron assimilation systems as a response to iron-limiting conditions encountered in environment and host's body fluids. Production of siderophores, small nonproteinaceous molecules with extremely high affinity for iron (III), is one of the most successful and widely utilized strategies of iron assimilation (Merrell et al., 2003). Common components of both siderophore-dependent and host iron-binding protein-dependent iron acquisition systems are receptor proteins involved in binding of siderophores and interacting with the host ironbinding proteins. These large outer membrane proteins are responsible for the transport of iron or iron-containing 
Table 7Consensus epitopes identified in H. pylori strain Hp26695

\begin{tabular}{|c|c|c|c|}
\hline Antigen No & Antigen Sequence & Epitope No & Consensus Epitopes \\
\hline AG2 & YESLMIDIVLVFNKGVAFSLLSFLEGGLKYLQILLILGLFIFLM & EPC5 & LLILGLFIF \\
\hline \multirow{2}{*}{ AG4 } & \multirow{2}{*}{ ALKSKAFRVSIQWNALVRKLLALE } & EPC37 & LVRKLLALE \\
\hline & & EPC38 & VRKLLALER \\
\hline AG6 & LRGFAYAFFTILFTLFLYAYIFSM & $\begin{array}{l}\text { EPC66 } \\
\text { EPC67 }\end{array}$ & $\begin{array}{l}\text { FLYAYIFSM } \\
\text { LFLYAYIFS }\end{array}$ \\
\hline AG8 & GNLLIVVILLCVAVFFTLKAIHIQK & EPC81 & FTLKAIHIQ \\
\hline AG19 & WMYSTFISLKTHLQFIE & EPC132 & LKTHLQFIE \\
\hline AG24 & FEEFQLHSLHLEV & EPC140 & FQLHSLHLE \\
\hline AG30 & IKAIGGLIIVGTCIYAY & EPC185 & LIIVGTCIY \\
\hline AG35 & SLIFKKVRIYSKMLVALGLSSVLIGCAM & EPC224 & FKKVRIYSK \\
\hline \multirow{2}{*}{ AG36 } & \multirow{2}{*}{ SSEHVTPLDFNYPIHIVQAPQNHHVVGILTPRIQVSDNLKPYID } & $\begin{array}{l}\text { EPC225 } \\
\text { EPC228 }\end{array}$ & $\begin{array}{l}\text { LIFKKVRIY } \\
\text { VVGILTPRI }\end{array}$ \\
\hline & & EPC229 & VGILTPRIQ \\
\hline AG41 & SNRVVHDFAVEVGT & EPC235 & VVHDFAVEV \\
\hline \multirow{2}{*}{ AG44 } & LDKYSLYYRLFNISSIVIGFLVALFSYGAGVILVYPILFLFALIIKPSFFYYTTYLLLLVSL & EPC262 & ILVYPILFL \\
\hline & SIISKYYLLSHA & EPC263 & VILVYPILF \\
\hline \multirow[t]{2}{*}{ AG46 } & HPEYIPCKVTCVTSG & ЕРС300 & YIPCKVTCV \\
\hline & & EPC337 & IFSANTLFL \\
\hline \multirow[t]{4}{*}{ AG53 } & IGSLVALLLGLPVLIFSANTLFLGAVFVGLIAIAQI & EPC338 & IGSLVALLL \\
\hline & & ЕРC339 & VALLLGLPV \\
\hline & & EPC346 & FFRIYDITK \\
\hline & & ЕРC347 & FRIYDITKP \\
\hline \multirow{4}{*}{ AG55 } & AMAISGLSLAGVILSFIFFRIYDITKPSLIGK & ЕРС 348 & FIFFRIYDI \\
\hline & AMAISULSLAGVILSFIFFKIY DIIKPSLIUK & ЕРC349 & ILSFIFFRI \\
\hline & & EPC350 & VILSFIFFR \\
\hline & & EPC351 & LSLAGVILS \\
\hline \multirow[t]{2}{*}{ AG58 } & AIPSKVIAIKDNVVLLETLGVQRE & EPC382 & VVLLETLGV \\
\hline & & EPC385 & LHIGYVMSK \\
\hline \multirow{3}{*}{ AG59 } & GESVKVGDYVLLHIGYVMSK & EPC386 & VLLHIGYVM \\
\hline & GESVKV UDY VLLFIUY VIMSK & EPC387 & YVLLHIGYV \\
\hline & & EPC388 & VGDYVLLHI \\
\hline AG73 & KERVSVPSK & EPC454 & KERVSVPS \\
\hline \multirow[t]{4}{*}{ AG74 } & & EPC464 & VSLVIVFC \\
\hline & VSLVIVFCCFLRAVELPGIY & EPC466 & IVFCCFLRA \\
\hline & & EPC467 & LVIVFCCFL \\
\hline & & EPC468 & VIVFCCFLR \\
\hline AG75 & TQEFLYMKSSFVEFF & EPC470 & YMKSSFVEF \\
\hline \multirow[t]{2}{*}{ AG76 } & KFYAYGISDV & EPC472 & FYAYGISDV \\
\hline & & EPC500 & FVLSSDHKI \\
\hline \multirow{3}{*}{ AG81 } & LNSL SVTKVECSKGKHHAYVFVI SSDHKUL SKI & EPC501 & YVFVLSSDH \\
\hline & & EPC502 & LSSDHKILS \\
\hline & & EPC503 & VLSSDHKIL \\
\hline \multirow[t]{3}{*}{ AG83 } & WFKCPKLSFVSDN & EPC505 & WFKCPKLSF \\
\hline & & EPC528 & FYFFAILTL \\
\hline & & EPC529 & ITVYVGAVI \\
\hline \multirow{4}{*}{ AG86 } & IAFYFFAILTLSMALVVITTTNILYAITALASSMVFISAFFFLLDAEFLGVVQITVYVGAV & EPC530 & VVQITVYVG \\
\hline & IVMYA & EPC531 & VYVGAVIVM \\
\hline & & EPC532 & LVVITTTNI \\
\hline & & EPC533 & VVITTTNIL \\
\hline AG88 & PKILCILSFGVALLLTLILSAPS & EPC540 & LTLILSAPS \\
\hline AG89 & DAQIPNIKAIGYVLFTNYLIPFEAAALMLLVAMVGGI & EPC542 & YVLFTNYLI \\
\hline AG92 & HIKVISI & EPC572 & HIKVISI \\
\hline
\end{tabular}

responsible for the transport of iron or iron-containing compounds throughthe otherwise impermeable outer membrane (Ye et al., 2003). Ferric transport system Exb B biopolymer transport protein in $H$. pylori is responsible for the transport of iron or iron-containing compounds through the impermeable outer membrane. Sequence analysis in E. coli, Haemophilusinfluenzae, Neisseria meningitides and Pseudomonas putida provided information on existence mechanism that utilizes Ton-independent heme. Knockout mutant and complementation studies in Neisseria meningitides established this fact (Sarangi et al., 2009). Designing an effective inhibitor to the existing multiple proteins for the utilization of heme-containing compounds effects the survival of $H$. pylori in their natural habitat, human mucosal surfaces.utilization of heme-containing compounds effects the

\section{Drug targets influencing RNA metabolism of the pathogen}

Ribonuclease $\mathrm{BN}$ is identified as a drug target in $H$. pylori. This drug target influences RNA metabolism of the pathogen. Ribonuclease, BN, lacking RNase $\mathrm{H}$ and RNase D activity was identified in $E$. coli and it is different from other exoribonucleases known till date in E. coli. RNase BN is a substrate specific with specificity towards $\mathrm{C}$-C-A sequence in tRNA than other types of tRNA and substrate specificity was proved both in vitro and in vivo. Mutants of these proteins affect the processing of tRNA's and ultimately synthesis of protein (Asha et al., 1983). Hence, an effective inhibitor for Ribonuclease BN can block the function of protein synthesis. 
Table 8 Vaccine Candidates for H. pylori strain Hp26695

\begin{tabular}{ccc}
\hline S. No & Protein ID & $\begin{array}{c}\text { Protein Name } \\
1\end{array}$ \\
NP_206874.1 & lipoprotein signal peptidase \\
2 & NP_206881.1 & Membrane Protein \\
3 & NP_206945.1 & Cbb3-type cytochrome c oxidase subunit Q CcoQ \\
4 & NP_206947.1 & DUF4006 superfamily \\
5 & NP_207125.1 & Flagellar protein FlaG \\
6 & NP_207241.1 & Transporter Protein \\
7 & NP_207289.1 & Putative paralog of $H p$ aA \\
8 & NP_207332.1 & cag pathogenicity island protein cag15 \\
9 & NP_207346.1 & 50S ribosomal protein L31 \\
10 & NP_207531.1 & Integral membrane protein \\
11 & NP_207692.1 & Hydrogenase expression/formation protein HypC \\
12 & NP_207808.1 & HtrA protease/chaperone protein / Serine protease \\
13 & NP_207818.1 & DUF2147 superfamily \\
14 & NP_207838.1 & Ribosome-binding factor A \\
15 & NP_208061.1 & NADH dehydrogenase subunit J \\
16 & NP_208233.1 & Carbon storage regulator \\
\hline
\end{tabular}

\section{Drug targets influencing respiration of the pathogen}

NADH-ubiquinone oxidoreductase chain $\mathrm{J}$ is identified as a drug target in $H$. pylori. This drug target influences metabolic pathway and effect respiration of the pathogen. The NADH ubiquinone oxidoreductase (Complex I), provides the input to the respiratory chain from the NAD-linked dehydrogenases of the citric acid cycle. The complex couples the oxidation of $\mathrm{NADH}$ and the reduction of ubiquinone, to the generation of a proton gradient which is then used for ATP synthesis. The complex occurs in the mitochondria of eukaryotes and in the plasma membranes of purple photosynthetic bacteria, and the closely related respiratory bacteria. All inhibitors affect the electron-transfer step from the high-potential iron-sulphur cluster to ubiquinone. Class I inhibitors appear to act directly at the ubiquinone-catalytic site which is related in complex I and glucose dehydrogenase (Friedrich.et al., 1994). Inhibitors designed to bind to NADH-ubiquinone oxidoreductase chain $\mathrm{J}$ competitively inhibit the protein from functioning which results in chemical asphyxiation of cells.

\section{Vaccine Candidates for $\mathrm{H}$. pylori}

Constructive screening protocol was implemented to identify suitable vaccine candidates for $H$. pylori. Choosing such a conservative way to face vaccine design inevitably implies missing some pathogen antigens, but still this is a small price to reach a valuable compromise. Forwarding further bioinformatics analyses on selected ones, may prove successful (Sandroet al., 2006). Bioinformatics approach has helped us in shortlisting and in identifying pathogenic factors from the proteome of the pathogen. Screening of pathogen factors for non-homology would shortlist the proteins which have the potential to cross react when vaccine is administered. Usually a protein has high probability of failure to cloning and express in experiment when it is likely to have more helices. Hence, proteins with alpha-helices $<3$ are selected for further analysis. (Sandroet al., 2006; Capecchiet al., 2004; Pizza et al., 2000). Screening of proteome for allergenicity would avoid the proteins which can elicit undesirable reaction during vaccination. Further, proteins showing positive predictions for antigenic, B-cell, T-cell activities are characterized as potential immunogens which are suitable for vaccine candidates.

\section{CONCLUSION}

Comparative genomics of $H$. pylori and Homosapien sapiens identified seven bacterial genes which are non-homologous to humans and are essential for pathogen. Four genes of the 7 predicted drug targets are already experimentally validated lending credence to our approach. These novel drug targets may have possible therapeutic implications for gastric cancer. Systematic insilico analysis approach identified 16 immunogenic proteins which are suitable vaccine candidates for $H$. pylori. Thus, bioinformatics approaches helped in rapid identification of novel drug targets and vaccine candidates for H. pylori strain Hp26695.

\section{Acknowledgements}

AMCP, DN and NNRR are thankful to the GITAM University, Visakhapatnam, India for providing the facility and support. AMCP, NNRR and DN are thankful to University Grants Commission, New Delhi for the project (UGC Project F.No.42$636 / 2013$ (SR) letter dated 25-03-2013). DN is thankful for the Project Fellowship sponsored by UGC, New Delhi, India. The authors also thankful to Professor IskaBhaskar Reddy and Professor Malla Rama Rao for constant support throughout the research work. We profusely thank Dr ChallaSurekha, GITAM University, Visakhapatnam, India for critical comments and reviewing of the manuscript.

\section{References}

Altschul, S.F., Gish, W., Miller, W., Myers, E.W. and Lipman, D.J. (1990): Basic local alignment search tool. J. Mol. Biol., 215(3): 403-410.

Arakawa, C., Kuratsu, M., Furihata, K., Hiratsuka, T., Itoh, N., Seto, H. and Dairi, T. (2011): Diversity of the early step of the futalosine pathway. Antimicrob. Agents Chemother., 55(2): 913-916.

Arora, S. and Czinn, S.J. (2005): Vaccination as a method of preventing Helicobacter pyloriassociated gastric cancer.CancerEpidemiol. Biomarkers Prev., 14: 18901891.

Asha, P.K., Blouin, R.T., Zaniewski, R. and Deutscher, M.P. (1983): Ribonuclease BN: Identification and partial characterization of a new tRNA processing enzyme. Proc. Natl. Acad. Sci. USA, 80: 3301-3304.

Ashok, K.T. (2013): CFSSP: Chou and Fasman secondary structure prediction server. Wide Spect., 1(9): 15-19.

Aziz, R.K., Bartels, D., Best, A.A., DeJongh, M., Disz, T., Edwards, R.A., Formsma, K., Gerdes, S., Lass, E.M., Kubal, M., Meyer, F., Olsen, G.J., Olson, R., Osterman, A.L., Overbeek, R.A., McNeil, L.K., Paarmann, D., Paczian, T., Parrello, B., Pusch, G.D., Reich, C., Stevens, R., Vassieva, O., Vonstein, V., Wilke, A. and Zagnitko, O. (2008): The RAST server: rapid annotations using subsystems technology. BMC Genomics, 9: 75.

Caffrey, C.R., Rohwer, A., Oellien, F., Marhöfer, R.J., Braschi, S., Oliveira, G., McKerrow, J.H. and Selzer, P.M. (2009): A comparative chemogenomics strategy to predict potential drug targets in the metazoan pathogen. Schistosomamansoni.PLoS ONE, 4(2): e4413.

Cai, J., Han, C., Hu, T., Zhang, J., Wu, D., Wang, F., Liu, Y., Ding, J., Chen, K., Yue, J., Shen, X. and Jiang, H. 
(2006): Peptide deformylase is a potential target for antiHelicobacter pylori drugs: reverse docking, enzymatic assay, and x-ray crystallography validation. Protein Sci., 15(9): 2071-2081.

Capecchi, B., Serruto, D., Adu-Bobie, J., Rappuoli, R. and Pizza, M. (2004): The genome revolution in vaccine research. Curr.Issues Mol. Biol., 1(6): 17-28.

Chou, P.Y. and Fasman, G.D. (1978): Empirical predictions of protein conformation.Annu. Rev. Biochem., 47: 251276.

Dorer, M.S., Fero, J. and Salama, N.R. (2010): DNA damage triggers genetic exchange in Helicobacter pylori. PLoSPathog., 6(7): e1001026.

Dutta, A., Singh, S.K., Ghosh, P., Mukherjee, R., Mitter, S. and Bandyopadhyay, D. (2006):Insilicoidentification of potential therapeutic targets in the human pathogen Helicobacter pylori. In Silico Biol., 6(2): 43-47.

Florez, A.F., Park, D., Bhak, J., Kim, B.C., Kuchinsky, A, Morris, J.H., Espinosa, J. and Muskus, C. (2010): Protein network prediction and topological analysis in Leishmania major as a tool for drug target selection. BMC Bioinformatics, 11(1): 484.

Friedrich, T., Heek, P., Leif, H., Ohnishi, T., Forche, E., Kunze, B., Jansen, R., Trowitzsch-Kienast, W., Höfle, G., Reichenbach, H. and Weiss, H. (1994): Two binding sites of inhibitors in NADH: ubiquinone oxidoreductase (complex I). Eur. J. Biochem., 219(1-2): 691-698.

Garg, A. and Gupta, D. (2008):VirulentPred: a SVM based prediction method for virulent proteins in bacterial pathogens.BMC Bioinformatics, 9(1): 62.

Garg, A. and Raghava, G.P.S. (2008): A machine learning based method for the prediction of secretory proteins using amino acid composition, their order and similaritysearch. In Silico Biol., 8(2): 129-140.

Graham, D.Y., Lew, G.M., Evans, D.G., Evans, D.J. and Klein, P.D. (1991): Effect of triple therapy (antibiotics plus bismuth) on duodenal ulcer healing. A randomized controlled trial. Ann. Intern. Med., 115(4): 266-269.

Heintschel V.H.E., Nalik, H.P. and Schmid, E.N. (1993): Characterisation of a Helicobacter pylori phage (HP1). J. Med. Microbiol., 38: 245-249.

Henikoff, S. and Henikoff, J.G. (1992):Amino acid substitution matrices from protein blocks. Proc. Natl. Acad. Sci. USA,89(22): 10915-10919.

Hossain, M.M., Mosnaz, A.T.M.J., Mohin, S.A., Kumar, P.R., Kabir, S.S., Ullah, S.M. and Prodhan, S.H. (2013): Identification of putative drug targets of Listeria monocytogenes $\mathrm{F} 2365$ by subtractive genomics approach. J. Biosci. Biotechnol.,2(5): 63-71.

Jehl, M.A., Arnold, R. and Rattei, T. (2011): Effective-a database of predicted secreted bacterial proteins. Nucleic Acids Res., 39: D591-D595.

Jensen, L.J., Gupta, R., Blom, N., Devos, D. Tamames, J., Kesmir, C., Nielsen, H., Stærfeldt, H.H., Rapacki, K., Workman, C. and Andersen, C.A. (2002): Prediction of human protein function from post-translational modifications and localization features. J. Mol. Biol., 319(5): 1257-1265.

Kolaskar, A.S. and Tongaonkar, P.C.(1990): A semiempirical method for prediction of antigenic determinants on protein antigens.FEBS Lett., 276(1-2): 172-4.

Manolov, A., Prihodko, E., Larin, A., Karpova, I., Semashko, T., Alexeev, D., Kostrjukova, E. and Govorun, V. (2014): Direct submission byBioinformatics, Research Institute for Physico-Chemical Medicine,MalayaPirogovskaya 1A, Moscow 119992, Russia. Availablefrom: http://www.ncbi.nlm.nih.gov/nuccore/GI:410024832.(A ccessedJuly 15, 2014).

Marchler-Bauer A. and Bryant S.H. (2004): CD-search: protein domain annotations on the fly. Nucleic Acids Res., 32(2): W327-31.

Maria, A., Scalab, E., Palazzob, P., Ridolfib, S., Zennarob, D. and Gabriele, C. (2006):Bioinformatics applied to allergy: allergen databases, from collecting sequence information to data integration. The Allergome platform as a model.Cell Immunol., 244(2): 97-100.

Merrell, D.S., Thompson, L.J., Kim, C.C., Mitchell, H., Tompkins, L.S., Lee, A. and Falkow, S. (2003): Growth phase-dependent response of Helicobacter pylori to iron starvation. Infect. Immun., 71(11): 6510-6525.

Molinari, M, Galli C, Norais N, Telford, J.L., Rappuoli, R. and Luzio, J.P. (1997):Vacuoles induced by Helicobacter pylori toxin contain both late endosomal and lysosomal markers. J. Biol. Chem., 272: 2533925344.

Nammi, D., Srimath-Tirumala-Peddinti, R.C.P.K. and Neelapu, N.R.R. (2016): Identification of drug targets in Helicobacter pylori by insilico analysis: possible therapeutic implications for gastric cancer. Curr. Cancer Drug Targets, 16(1): 79-98.

Neelapu N.R., Nammi, D., Pasupuleti, A.C., Surekha, C. (2014): Helicobacter pylori induced gastric inflammation, ulcer, and cancer: A pathogenesis perspective. Interdiscip. J. Microinflamm., 1(2): 113.

Neelapu, N.R.R. and Pavani, T. (2013): Identification of novel drug targets in $H p \mathrm{~B} 38, H p \mathrm{P} 12, H p \mathrm{G} 27, H p$ shi470, HpSJM180strains of Helicobacter pylori: An insilicoapproach for therapeutic intervention. Curr. Drug Targets, 14(5): 601-611.

Neelapu, N.R.R., Mutha, V.R.N. and Akula, S. (2015): Identification of potential drug targets in Helicobacter pylori strain HpAG1 by insilico genome analysis. Infect. Disorders Drug Targets, 15(2): 106-117.

Neelapu, N.R.R., Nammi, D., Pasupuleti, A.M.C. and Challa, S. (2016): Targets against Helicobacter pylori and other tumor-producing bacteria. In: Villa T.G. and Vinas M. (eds) New Weapons to Control Bacterial Growth, Springer International Publishing, pp. 239-279.

Neelapu, N.R.R.,Srimath-Tirumala-Peddinti, R.C.P.K., Deepthi, N. and Pasupuleti, A.C.M (2013): New strategies and paradigm for drug target discovery: a special focus on infectious diseases tuberculosis, malaria, leishmaniasis, trypanosomiasis and gastritis. Infect. Disorders Drug Targets, 13(5): 352-364.

Pizza, M., Scarlato, V., Masignani, V., Giuliani, M.M., Arico, B., Comanducci, M., Jennings, G.T., Baldi, L., Bartolini, E., Capecchi, B., Galeotti, C.L., Luzzi, E., Manetti, R., Marchetti, E., Mora, M., Nuti, S., Ratti, G., Santini, L., Savino, S., Scarselli, M., Storni, E., Zuo, P., 
Broeker, M., Hundt, E., Knapp, B., Blair, E., Mason, T., Tettelin, H., Hood, D.W., Jeffries, A.C., Saunders, N.J., Granoff, D.M., Venter, J.C., Moxon, E.R., Grandi, G. and Rappuoli, R.(2000):Identification of vaccine candidates against serogroup B meningococcus by whole-genome sequencing. Science, 287(5459): 18161820 .

Rauws, E.A. and Tytgat, G.N. (1990): Cure of duodenal ulcer associated with eradication of Helicobacter pylori. Lancet, 335(8700): 1233-1235.

Reyrat, J.M., Pelicic, V., Papini, E., Montecucco, C., Rappuoli, R. and Telford, J.L. (1999): Towards deciphering the Helicobacter pylori cytotoxin. Mol. Microbiol., 34: 197-204.

Saha, S. and Raghava G.P.S. (2006): Prediction of continuous B-cell epitopes in an antigen using recurrent neural network. Proteins, 65(1): 40-48.

Saha, S. and Raghava, G.P.S. (2006): VICMpred: SVMBased method for the prediction of functional proteins of gram-negative bacteria using amino acid patterns and composition. Geno. Prot. Bioinfo., 4(1): 42-47.

Saha, S. and Raghava, G.P.S. (2007): Prediction of bacterial proteins. In Silico Biol., 7(5): 0028.

Saha, S., Zack, J., Singh, B. and Raghava, G.P.S. (2007): VGIchan: Prediction and classification of voltage-gated ion channels. Geno. Prot. Bioinfo., 4(4): 253-258.

Sakharkar, K.R., Sakharkar, M.K. and Chow, V.T.K. (2004): A novel genomics approach for the identification of drug targets in parasites, with special reference to Pseudomonas aeruginosa. In Silico Biol., 4(3): 355-360.

Sandro, V., Filippo, B. and Francesco, F. (2006): Nerve: new enhanced reverse vaccinology environment. $B M C$ Biotechnol.,6: 35.

Sarangi, A.N., Aggarwal, R., Rahman, Q. and Trivedi, N. (2009): Subtractive genomics approach for insilicoidentification and characterization of novel drug targets in Neisseria meningitidisserogroup B. J. Comput. Sci. Syst. Biol., 2(5): 255-258.
Sarkar, M., Maganti, L., Ghoshal, N. and Dutta C. (2012): Insilico quest for putative drug targets in Helicobacter pyloriHpAG1: Molecular modeling of candidate enzymes from lipopolysaccharide biosynthesis pathway. J. Mol. Model., 8(5): 1855-1866.

Suthar, N., Goyal, A. and Dubey, V.K. (2009): Identification of potential drug targets of Leishmaniainfantumby insilicogenome analysis. Lett.Drug Des.Discov., 6(8): 620-622.

Uddin, R. and Saeed, K. (2014): Identification and characterization of potential drug targets by subtractive genome analyses of methicillin resistant Staphylococcus aureus. Comput. Biol. Chem., 63(4): 48-55.

Weinberg, M.V. and Maier, R.J. (2007): Peptide transport in Helicobacter pylori: roles of Dpp and Opp systems and evidence for additional peptide transporters. $J$. Bacteriol., 189(9): 3392-3402.

Wiwanitkit, V. (2005): Analysis of Mycobacterium lepreagenome: Insilicosearching for drug targets. Southeast Asian J. Trop. Med. Public Health, 36: 225227.

Ye, S., Von Delft, F., Brooun, A., Knuth, M.W., Swanson. R.V. and McRee, D.E. (2003): The crystal structure of shikimate dehydrogenase (AroE) reveals a unique NADPH binding mode. J. Bacteriol., 185(14): 41444151.

Yu, C.S., Lin, C.J. and Hwang, J.K. (2004):Predicting subcellular localization of proteins for Gram-negative bacteria by support vector machines based on $n$-peptide compositions. Protein Sci. J.,13: 1402-1406.

Zhang, Z.W. (1994): Schistosomes. liver flukes and Helicobacter pylori. IARC working group on the evaluation of carcinogenic risks to humans. Lyon, 7-14 June 1994. IARC Monogr.Eval.Carcinog. Risks Hum., 61:1-241.

\section{How to cite this article:}

Amita Martin Corolinaet al.2017, Screening And Identification of Drug Targets And Vaccine Candidates For Helicobacter Pylori Strain Hp26695.Int J Recent Sci Res. 8(4), pp.16384-16395.DOI: http://dx.doi.org/10.24327/ijrsr.2017.0804.0140 